https://doi.org/10.22319/rmcp.v12i1.5131

Artículo

\title{
Actividad antihelmíntica in vivo de hojas de Acacia cochliacantha sobre Haemonchus contortus en cabritos Boer
}

Gastón Federico Castillo-Mitre ${ }^{a}$

Rolando Rojo-Rubio ${ }^{\text {a }}$

Agustín Olmedo-Juárez ${ }^{\text {b* }}$

Pedro Mendoza de Gives ${ }^{b}$

José Fernando Vázquez-Armijo ${ }^{\text {a }}$

Alejandro Zamilpa ${ }^{c}$

Héctor Aarón Lee-Rangel d

Leonel Avendaño-Reyes ${ }^{\mathrm{e}}$

Ulises Macias-Cruz ${ }^{\mathrm{e}}$

${ }^{a}$ Universidad Autónoma del Estado de México, Centro Universitario UAEM-Temascaltepec.

b Instituto Nacional de Investigaciones Forestales Agrícolas y Pecuarias, Centro Nacional de Investigación en Disciplinaria en Salud Animal e Inocuidad, carretera Federal Cuernavaca, Cuautla No. 8534/ Col. Progreso. 62550 Jiutepec, Morelos/A.P. 206-CIVAC, México.

${ }^{\mathrm{c}}$ Instituto Mexicano del Seguro Social. Centro de Investigación Biomédica del Sur. México.

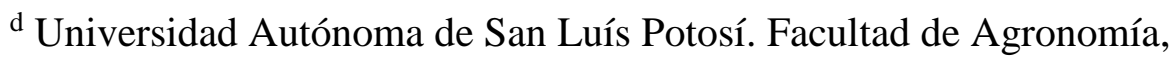

e Universidad Autónoma de Baja California. Instituto de Ciencias Agrícolas,

*Autor de correspondencia: olmedo.agustin@inifap.gob.mx; aolmedoj@gmail.com 


\section{Resumen:}

El objetivo del presente trabajo fue evaluar el efecto de la inclusión de hojas de Acacia cochliacantha en una dieta de mantenimiento para cabritos de la raza Boer en el conteo fecal de huevos (CFH) de Haemonchus contortus, consumo de agua y materia seca. Diez cabritos recién destetados (16.850 $\pm 1.630 \mathrm{~kg}$ de peso vivo inicial y 3 meses de edad), fueron experimentalmente infestados con larvas (L3) de H. contortus (350 larvas por kilogramo de peso vivo), para probar dos tratamientos: T1: testigo (animales infectados con larvas L3 de H. contortus, sin inclusión de hojas de $A$. cochliacantha) y T2: animales infectados con larvas L3 de H. contortus, con inclusión de $5 \%$ hojas de $A$. cochliacantha en la dieta. Antes de asignar los tratamientos a los animales, se realizó un $\mathrm{CFH}$ por gramos de heces en cada uno de ellos y de esta manera agruparlos de mayor a menor en relación a la cantidad de huevos presentes en las heces, en los dos animales con valores más altos se asignaron aleatoriamente T1 o T2 y así sucesivamente hasta completar cinco repeticiones verdaderas para cada tratamiento. Las variables medidas fueron: CFH (por gramo de heces), consumo de agua y consumo de materia seca (CMS). Los resultados encontrados demostraron reducción $(P<0.05)$ en el CFH de H. contortus en los cabritos donde se incluyó el $5 \%$ de hojas de $A$. cochliacantha en su dieta. Para el consumo de agua y CMS ( $\mathrm{g} \mathrm{día}^{-1}$ ) no existieron diferencias entre tratamientos $(P>0.05)$. Se concluye que la adición de hojas de $A$. cochliacantha en dietas para cabritos tienen actividad antihelmíntica, por lo que esta leguminosa arbórea podría representar una opción en el manejo integral de la nematodiasis de cabritos Boer en crecimiento.

Palabras clave: Cabritos, Antihelmíntico, Acacia cochliacantha, Haemonchus contortus.

Recibido: $28 / 10 / 2018$

Aceptado: 28/10/2019

\section{Introducción}

Las enfermedades causadas por los nematodos gastrointestinales (NGI) en los pequeños rumiantes representan uno de los mayores problemas en el mundo, ya que afectan su salud y productividad. Haemonchus contortus es el principal NGI, que ocasiona las mayores pérdidas económicas en la industria ganadera en México, se estima que este problema puede representar hasta 445.1 millones de dólares anuales ${ }^{(1-3)}$. En las últimas décadas, el uso indiscriminado de antiparasitarios para el control de NGI, ha ocasionado resistencia antihelmíntica; además del efecto residual que tienen algunos fármacos en el organismo y el ambiente ${ }^{(4,5)}$. Esta problemática se está investigando para proponer alternativas de control sostenibles, focalizándose en el estudio de los metabolitos secundarios de las plantas leguminosas con propiedades antihelmínticas ${ }^{(6-9)}$. Dentro de dichos 
compuestos se tienen a los taninos condensados, terpenos, saponinas y flavonoides ${ }^{(10)}$. De manera particular se ha demostrado que los flavonoides presentan actividad nematicida, que junto con taninos condensados libres actúan sinérgicamente potenciando su efecto contra los $\mathrm{NGI}^{(11,12)}$. Acacia cochliacantha es una leguminosa conocida como "cubata" que se ha utilizado en la medicina tradicional para el tratamiento de enfermedades gastrointestinales ${ }^{(13)}$. Diversos trabajos han reportado actividad nematicida in vitro a partir de extractos de sus hojas ${ }^{(8,12)}$, o compuestos específicos como los taninos, quercetina, ácido cafeico, cumárico y ferúlico ${ }^{(12)}$. El objetivo del presente trabajo fue evaluar el efecto de la adición de hojas de A. cochliacantha en la dieta de cabritos Boer en crecimiento infectados experimentalmente con $H$. contortus, sobre la eliminación de huevos, consumo de materia seca y agua.

\section{Material y métodos}

\section{Zona de estudio}

El presente trabajo se desarrolló en la unidad experimental de pequeños rumiantes del Centro Universitario UAEM Temascaltepec; este lugar presenta una altura media sobre el nivel del mar de $1,740 \mathrm{~m}$, con un clima del tipo cálido sub-húmedo con lluvias en verano $(\mathrm{Aw})^{(14)}$.

\section{Material vegetal}

Se recolectaron hojas frescas (jóvenes y maduras) de Acacia cochliacantha en la localidad "El Salitre Palmarillos", municipio de Amatepec, Estado de México, México, cuya localización está a $18^{\circ} 43^{\prime} 28^{\prime \prime} \mathrm{N}$ y $100^{\circ} 17^{\prime} 03^{\prime \prime} \mathrm{O}$. El material vegetativo se colectó entre marzo y abril de 2016, durante las primeras horas de la mañana; las hojas fueron depositadas en una hielera para evitar cambios en su estructura química por efectos de fotoxidación, posteriormente se transportaron al laboratorio de nutrición animal del Centro Universitario UAEM Temascaltepec, donde se seleccionó un ejemplar para su identificación en el Herbario Nacional de México de la Universidad Nacional Autónoma de México. El material vegetal fue identificado por el Prof. Rafael Torres-Colín y depositado en el Herbario Nacional bajo el código de colecta OD07042016. Las hojas frescas se secaron a temperatura ambiente bajo la sombra hasta alcanzar peso constante, y así molerlas a un tamaño de partícula de 4 a 6 mm utilizando un molino eléctrico Wiley Mod. TS3375E15.

\section{Análisis químicos}

Se realizó análisis bromatológico de las dietas experimentales y hojas de A. cochliacantha utilizando los siguientes métodos: materia seca (MS), materia orgánica (MO), proteína cruda (PC), extracto etéreo $(\mathrm{EE})^{(15)}$; para el fraccionamiento de la pared celular (FDN y FDA) se utilizó la 
metodología descrita por Van Soest et $a l^{(16)}$. El porcentaje de inclusión de las hojas de $A$. cochliacantha se le determinó el contenido de taninos condensados totales (TCT), de acuerdo a la técnica descrita por Terrill et $a l^{(17)}$, modificada por López et al $l^{(18)}$; taninos condensados ligados a proteína y a fibra se utilizando el método de Porter et $a l^{(19)}$ con las modificaciones realizadas por Hagerman $^{(20)}$.

\section{Material biológico}

Se obtuvieron larvas infectantes de Haemonchus contortus (L3, cepa INIFAP) a partir de un ovino donador (22.3 $\pm 0.5 \mathrm{~kg}$ de peso vivo) infectado con una dosis oral única de $350 \mathrm{~L} 3$ por kilo de peso vivo. El cordero fue atendido de acuerdo a la norma (NOM-062-ZOO-1999). Las larvas infectantes de $H$. contortus se obtuvieron a partir de cultivos mediante la técnica de Baermann ${ }^{(21)}$.

\section{Animales experimentales}

Se utilizaron catorce cabritos machos de la raza Boer $(16.850 \pm 1.6 \mathrm{~kg}$ de peso vivo y 3 meses de edad) procedentes del rancho el Salitre, perteneciente al Centro Universitario UAEM Temascaltepec. Antes de iniciar el experimento, todos los animales se desparasitaron con ivermectina comercial a una dosis única de $0.22 \mathrm{mg}^{-1} \mathrm{~kg}$ de peso vivo, esto con la finalidad de eliminar cualquier infección natural de NGI. Después de aplicar el antiparasitario, los cabritos se alojaron en corraletas individuales (equipados con sombra, comedero y bebedero). Pasado ese periodo, se corroboró que los animales estuvieran negativos a infección de NGI mediante la técnica de McMaster $^{(22)}$ y al día 16 se inocularon experimentalmente de forma individual con una dosis única de $350 \mathrm{~L} 3$ por $\mathrm{Kg}$ de peso vivo de $H$. contortus. Una vez que los cabritos fueron diagnosticados como positivos a la presencia de huevos de $H$. contortus (día 30), se lotificaron en dos grupos $(n=7)$ acorde a su eliminación de huevos por gramo de heces (HPG) y a su peso corporal. Previo a la infección, los animales recibieron una dieta de adaptación sin hojas de $A$. cochliacantha (Cuadro 1). Las dietas se balancearon basándose a los requerimientos para cabritos en crecimiento mediante las tablas del NRC del 2007 para pequeños rumiantes ${ }^{(23)}$. 
Cuadro 1: Ingredientes, aporte proteína y energía metabolizable de las dietas experimentales de mantenimiento proporcionadas a cabritos infectados con Haemonchus contortus

\begin{tabular}{lll}
\hline \multicolumn{1}{c}{ Ingrediente (\%) } & Tratamiento & \\
\hline Acacia cochliacantha & 1 & 2 \\
Heno de alfalfa & 0.00 & 5.00 \\
Maíz molido & 33.00 & 28.00 \\
Sorgo molido & 5.00 & 5.00 \\
Pasta de soya & 17.50 & 17.50 \\
Salvado de trigo & 7.00 & 7.00 \\
Paja de avena & 10.00 & 10.00 \\
Melaza & 25.00 & 25.00 \\
Premezcla de vitaminas y minerales & 0.00 & 0.00 \\
Total & 2.50 & 2.50 \\
Aporte de proteína cruda & 100.00 & 100.00 \\
Aporte de energía metabolizable (MJ/kg MS) & 12.78 & 12.76 \\
\hline T: & 2.84 & 2.83 \\
\hline
\end{tabular}

T1: control (animales infectados, sin inclusión de hojas de A. cochliacantha) y T2: animales infectados, con inclusión de $5 \%$ hojas de $A$. cochliacantha en la dieta.

${ }^{1}$ Calculado de acuerdo a los contenidos individuales de energía de cada alimento incluido en la dieta ${ }^{(23) .}$

\section{Diseño experimental}

Durante el desarrollo del experimento y debido a que los cabritos estaban bajo un estrés pos destete, se puso en peligro la vida de cuatro de ellos, ya que los valores de volumen celular aglomerado (VCA) eran muy bajos, lo que indicó un estado grave de anemia, y los conteos fecales de huevos eran demasiados altos (HPG > 12,500), por lo que fue necesario retirarlos del experimento, quedando solo 10 de ellos, los cuales se asignaron a los dos tratamientos previamente establecidos: T1: control (animales infectados con larvas L3 de H. contortus y sin adición de hojas de $A$. cochliacantha en su dieta) y T2: tratamiento (animales infectados con larvas L3 de $H$. contortus y adición de 5\% hojas de A. cochliacantha en su dieta). Las variables de respuesta fueron: CFH por gramo de heces, consumo de materia seca y agua. Semanalmente se tomaron muestras de heces (días pos-infección: 1, 7, 14, 21 y 28) directamente del recto de cada animal. El material fecal se procesó para estimar el CFH por gramo de heces utilizando la técnica de $\mathrm{McMaster}^{(22)}$. El consumo de materia seca y agua se determinó diariamente mediante la sustracción del alimento o agua ofrecida menos el rechazo. La eficacia antiparasitaria fue estimada mediante la fórmula de Abbott:

Eficacia (reducción de $\mathrm{CFH} g$ heces, \%) $=[(\mathrm{CFH} g$ heces del grupo control $-\mathrm{CFH} g$ heces de grupo tratado) / CFH g heces del grupo control)]*100 


\section{Análisis estadístico}

Los resultados se procesaron mediante un análisis de varianza utilizando el procedimiento GLM del $\mathrm{SAS}^{(24)}$. Bajo un diseño completamente al azar, cuando existieron diferencias entre tratamientos, las medias se separaron mediante la prueba PDIFF, STDERR statement. La significancia se declaró a un nivel de $P \leq 0.05$ y la tendencia de $0.05<P \leq 0.10^{(25)}$.

\section{Resultados}

\section{Análisis químico}

Los resultados de la composición química de las dietas experimentales, así como el contenido de taninos en las hojas de A. cochliacantha se muestran en los Cuadros 1 y 2.

Cuadro 2: Análisis químico proximal de hojas deshidratadas de Acacia cochliacantha y su contenido de taninos

\begin{tabular}{lllllllll}
\hline & MO & PC & FDN & FDA & TCL & TCP & TCF & TCT \\
\cline { 2 - 7 } $\begin{array}{l}\text { A. } \\
\text { cochliacantha }\end{array}$ & 937.0 & 163.0 & 698.6 & 581.7 & 140.0 & 26.0 & 36.0 & 202.0 \\
\hline $\begin{array}{l}\text { MO= materia orgánica, PC= proteína cruda, FDN= fibra detergente neutro, FDA= fibra detergente acido, TCL= } \\
\text { taninos condensados libres, TCP= taninos condensados ligados a proteína, TCF= taninos condensados ligados a } \\
\text { fibra, TCT }=\text { taninos condensados totales. }\end{array}$
\end{tabular}

\section{Conteo fecal de huevos}

Los resultados del CFH por gramo de heces y porcentaje de eficacia de la dieta que contenía hojas deshidratadas de A. cochliacantha se presentan en el Cuadro 3. El porcentaje de reducción causado por esta leguminosa estuvo en intervalos de 32.8-70.8 a partir del día 14 al 28 respectivamente. 
Cuadro 3: Conteo fecal de huevos de Haemonchus contortus $(\mathrm{CFH}$, media \pm desviación estándar) y porcentaje de eficacia de la dieta adicionada con hojas de Acacia cochliacantha

\begin{tabular}{|c|c|c|c|c|c|c|}
\hline \multirow[b]{2}{*}{ Tratamiento } & & \multicolumn{5}{|c|}{ Días de muestreo } \\
\hline & & 1 & 7 & 14 & 21 & 28 \\
\hline \multirow[t]{3}{*}{$\mathrm{T} 1$} & $\begin{array}{l}\mathrm{CFH}(/ \mathrm{g} \\
\text { de heces) }\end{array}$ & $1100 \pm 120$ & $3250 \pm 735^{\mathrm{b}}$ & $2980 \pm 345^{\mathrm{a}}$ & $5300 \pm 435^{\mathrm{a}}$ & $6850 \pm 1432.90^{\mathrm{a}}$ \\
\hline & $\%$ & ---- & ---- & ---- & ---- & ---- \\
\hline & Eficacia & & & & & \\
\hline \multirow[t]{2}{*}{$\mathrm{T} 2$} & $\begin{array}{l}\mathrm{CFH}(/ \mathrm{g} \\
\text { de heces) }\end{array}$ & $1100 \pm 133$ & $6850 \pm 828^{a}$ & $2000 \pm 367^{b}$ & $2050 \pm 432^{b}$ & $2000 \pm 178^{b}$ \\
\hline & $\begin{array}{l}\% \\
\text { Eficacia }\end{array}$ & ---- & -110.7 & 32.8 & 81.1 & 70.8 \\
\hline Valor de $P$ & & $>0.15$ & $<0.05$ & $<0.01$ & $<0.01$ & $<0.01$ \\
\hline
\end{tabular}

T1 = testigo (animales infectados con larvas L3 de H. contortus) y T2= animales infectados con larvas L3 de $H$. contortus, con inclusión de 5\% hojas de A. cochliacantha en la dieta.

\section{Consumo de agua y materia seca}

Los resultados de consumo de agua y materia seca son mostrados en el Cuadro 4. No se encontró $(P>0.05)$ efecto en ambas variables.

Cuadro 4: Consumo de agua (CA) y materia seca (MS) por día en cabritos infectados con Haemonchus contortus recibiendo una dieta adicionada con hojas de Acacia cochliacantha

\begin{tabular}{|c|c|c|c|c|c|c|c|c|c|c|}
\hline \multicolumn{11}{|c|}{ Días de muestreo } \\
\hline & 1 & & 7 & & 14 & & 21 & & 28 & \\
\hline $\mathrm{T}$ & $\mathrm{CA}$ & MS & $\mathrm{CA}$ & MS & $\mathrm{CA}$ & MS & $\mathrm{CA}$ & MS & $\mathrm{CA}$ & MS \\
\hline 1 & $1.7 \pm 0$ & $436.3 \pm 47$ & $1.6 \pm 0$. & $530.0 \pm 80$ & $1.8 \pm 0$. & $650.4 \pm 17$ & $1.7 \pm 0$ & $647.7 \pm 20$ & $1.8 \pm 0$. & $651.5 \pm 21$ \\
\hline & 3 & 6 & 1 & 4 & 1 & 3.2 & 08 & 6.7 & 2 & 1.0 \\
\hline 2 & $1.6 \pm 0$. & $448.7 \pm 10$ & $1.5 \pm 0$. & $559.7 \pm 14$ & $1.8 \pm 0$. & $625.0 \pm 21$ & $1.7 \pm 0$. & $745.1 \pm 27$ & $1.7 \pm 0$. & $694.5 \pm 19$ \\
\hline & 3 & 0.0 & 4 & 1.3 & 5 & 6.6 & 4 & 2.5 & 4 & 4.2 \\
\hline$P$ & 0.71 & 0.80 & 0.69 & 0.69 & 0.76 & 0.84 & 0.90 & 0.52 & 0.75 & 0.76 \\
\hline
\end{tabular}

\section{Discusión}

El presente estudio mostró efecto antiparasitario atribuido al consumo de hojas de $A$. cochliacantha en los cabritos, de tal forma que el consumo prolongado de esta leguminosa tiene beneficios sobre 
la salud de los animales. Recientemente esta leguminosa fue evaluada contra parásitos gastrointestinales de bovinos y ovinos bajo condiciones in vitro, y se encontraron resultados positivos $^{(8,12)}$. En esos trabajos se demostró la actividad ovicida y larvicida en contra de diferentes NGI, entre ellos a Haemonchus contortus. Con estos antecedentes, la presente investigación confirma el efecto antiparasitario in vivo contra los nematodos gastrointestinales de mayor importancia en la salud de los pequeños rumiantes.

Estos mismos resultados han sido reportados en investigaciones previas, cuando la inclusión de hojas deshidratadas de Lysiloma acapulcensis en la dieta de ovinos de pelo que fueron infectados experimentalmente con larvas (L3) de H. contortus, disminuyó en $67.7 \%$ la excreción fecal de huevos por gramo de heces ${ }^{(9)}$. En otro trabajo realizado en Brasil se reportaron reducciones hasta del $70 \%$ al suministrar hojas de Mimosa caesalpiniifolia en ovinos infectados con H. contortus $^{(26)}$.

Por otra parte, es sabido que, cuando arboles leguminosos forman parte de la dieta de los rumiantes, no solo se obtendrán beneficios en la salud, sino que también se incrementa la ingesta de proteína dietaría, dado que la mayoría de estos recursos forrajeros contienen más del $18 \%$ de PC en su materia orgánica, concentraciones altas si son comparadas con aquellas de las gramíneas que raramente sobrepasan el $10 \%$, con esto queda de manifiesto que, estás plantas tienen un efecto nutraceútico en los animales ${ }^{(27)}$. En este sentido, existen diversas investigaciones que han hipotetizado que animales alimentados a base de gramíneas y leguminosas, la proporción de consumo dependerá del nivel de proteína en las gramíneas. Y si éste llegara a ser menor a $8 \%$, los animales incrementarán su consumo de leguminosas, las cuales pueden ser arbustivas y arbóreas. Al respecto, en un estudio realizado por Méndez-Ortíz et al ${ }^{(28)}$ observaron que ovinos infectados por $H$. contortus consumían mayor cantidad de una leguminosa arbórea rica en metabolitos secundarios (Havardia albicans). Sin embargo, cuando este tipo de leguminosas son suministradas en las dietas de los animales en confinamiento, los efectos pueden ser positivos o negativos, afectando directamente el nivel de ingesta alimenticia y proteína metabolizable, lo cual está relacionado directamente con la cantidad de taninos condensados por gramo de materia seca. Se menciona que para obtener beneficios, la cantidad de taninos condensados no debería sobrepasar el $5 \%$ del total de la dieta ${ }^{(29)}$.

En el presente estudio no se observaron efectos negativos en el consumo de agua y materia seca en los animales que estuvieron consumiendo la dieta adicionada con hojas de A. cochliacantha, lo cual demostró que la ingesta de taninos condensados totales no afectó el consumo de materia seca. De acuerdo a lo reportado en la literatura, se menciona que cuando la dieta contiene altos niveles de compuestos secundarios y de manera particular taninos condensados libres (superiores a los 50 $\mathrm{g} \mathrm{kg}^{-1}$ de MS), se podría inhibir la actividad microbiana afectando la digestibilidad de la fracción potencialmente digestible de los nutrientes y por consecuencia deprimir el consumo de materia seca al aumentar el tiempo medio de retención en el rumen ${ }^{(30)}$. Aunque este fenómeno podría tener 
un efecto positivo en el metabolismo de los compuestos nitrogenados al disminuir la proteólisis ruminal, lo que derivaría en un aumento de la proteína de sobrepaso e incrementar el flujo de aminoácidos a duodeno, aumentando el conjunto de proteína metabolizable para fines productivos en los animales ${ }^{(31)}$.

Los resultados del análisis en taninos condesados presentes en A. cochliacantha, (Cuadro 2) revelaron que es una planta que podría tener efectos como los explicados anteriormente ya que tiene $20.2 \%$ de taninos condensados totales y $14.0 \%$ de taninos condensados libres (TCL), los cuales son los compuestos bioactivos y pueden secuestrar a los carbohidratos y proteínas de la dieta. Sin embargo, debido a que sólo se incluyó el $5 \%$ de las hojas de A. cochliacantha en la dieta total el consumo de taninos condensados totales (TCT) fue el $5.70 \mathrm{~g}$ y de estos solo sólo $3.9 \mathrm{~g}$ fueron de TCL, concentración que no fue suficiente para deprimir el consumo de materia seca en los animales que se les incluyó hojas de A. cochliacantha en su dieta. Estos resultados están relacionados con un estudio realizado por León-Castro et al ${ }^{(32)}$ donde reportaron importantes reducciones de HPG de $H$. contortus en cabritos infectados de forma artificial que estuvieron alimentados con un $10 \%$ de inclusión de follaje de $A$. cochliacantha sin observar efectos negativos en la salud de los animales.

No obstante, futuros estudios serán considerados como la dosis respuesta sobre el consumo, digestibilidad de la materia seca y orgánica de dietas basales con diferentes niveles de hojas de $A$. cochliacantha. Se conoce que las plantas ricas en metabolitos secundarios como los taninos contienen propiedades nutracéuticas ${ }^{(7)}$ y en muchos trabajos de nutrición animal se ha comprobado que los taninos son los poli-fenoles responsables de mejorar el valor nutricional de las dietas para el ganado. Del mismo modo, se ha reportado que extractos de plantas ricas en taninos pueden contener propiedades antiparasitarias ${ }^{(32-35)}$. Sin embargo, pese a que $A$. cochliacantha contiene altas cantidades de taninos condensados se podría inferir que estos compuestos son los probables responsables de la actividad antiparasitaria; no obstante Castillo-Mitre et $a l^{(12)}$, reportaron que los compuestos responsables de la actividad fueron derivados del ácido hidroxicináminco (ácido cafeico, cumárico, ferúlico y metil cafeatos). Por lo tanto, la actividad antiparasitaria de esta leguminosa podría deberse a estos compuestos y los taninos podrían colaborar de forma secundaria sobre el control de $H$. contortus mediante la protección de proteína de la dieta a los microorganismos del rumen. En este sentido, los resultados del presente trabajo (in vivo) y los de otros estudios in vitro ${ }^{(12)}$ con $A$. cochliacantha podrían ser importantes para realizar futuras investigaciones sobre sus propiedades nutracéuticas en pequeños rumiantes. 


\section{Conclusiones e implicaciones}

Los resultados del presente trabajo muestran que Acacia cochliacantha contiene propiedades antiparasitarias y puede ser una fuente de alimento importante para cabritos infectados con NGI, ya que no se deprime el consumo de agua y alimento.

\section{Conflicto de intereses}

Los autores declaran que no existe conflicto de intereses.

\section{Agradecimientos}

Parte de este trabajo fue financiado por INIFAP (proyecto SIGI 8215734475) y la Universidad Autónoma del Estado de México (Proyecto UAEM 4585/2018/CIP) y la Red Temática de Farmoquímicos, CONACYT (Proyecto número 294727, 2018). Este estudio fue parte de tesis doctoral de Gastón Federico Castillo-Mitre bajo la dirección del Dr. Rolando Rojo-Rubio y el Dr. Agustín Olmedo-Juárez.

\section{Literatura citada:}

1. Rodíguez-Vivas RI, Grisi L, Pérez-de León AA, Silva-Villela H, Torres-Acosta JFJ, FragosoSánchez H. et al. Evaluación del impacto económico potencial de los parásitos del ganado bovino en México. Revisión. Rev Mex Cienc Pecu 2017; 8(1):61-74.

2. Marume U, Chimonyo M, Dzama K. A preliminary study on the responses to experimental Haemonchus contortus infection in indigenous goat genotypes. Small Ruminant Res 2011; 95:70-74.

3. Githiori J, Athanasiadou S, Thamsbprg S. Use of plants in novel approaches for control of gastrointestinal helminthes in livestock with emphasis on small ruminants. Vet Parasitol 2006;139:308-320.

4. Jackson F, Coop RL. The development of anthelmintic resistance in sheep nematodes. Parasitol 2000;120:95-107.

5. Tsiboukis D, Sazakli E, Jelastopulu E, Leotsinidis M. Anthelmintics residues in raw milk. Assessing intake by a children population. Pol J Vet Sci 2013;16:85-91.

6. Olmedo-Juárez A, Rojo-Rubio R, Arece-García J, Mohamed AZS, Kholif EA, Morales AE. In vitro of Pithecellobium dulce and Lysiloma acapulcensis on the exogenous development of gastrointestinal strongyles in sheep. Ital J Anim Sci 2014;13:303-307. 
7. García-Hernández C, Arece-García J, Rojo-Rubio R, Mendoza-Martínez GD, Albarrán-Portillo B, Vázquez-Armijo JF, et al. Nutraceutic effect of free condensed tannins of Lysiloma acapulcensis (Kunth) Benth on parasite infection and performance of Pelibuey sheep. Trop Anim Health Prod 2017;49:55-61.

8. Olmedo-Juárez A, Rojo-Rubio R, Zamilpa A, Mendoza-de Gives P, Arece-García J, LópezArellano ME, et al. In vitro larvicidal effect of a hydroalcoholic extract from Acacia cochliacantha leaf against ruminant parasitic nematodes. Vet Res Commun 2017;41:227-232.

9. González-Cortazar M, Zamilpa A, López-Arellano ME, Aguilar-Marcelino L, Reyes-Guerrero DE, Olazarán-Jenkins S, et al. Lysiloma acapulcensis leaves contain anthelmintic metabolites that reduce the gastrointestinal nematode egg population in sheep faeces. Comp Clin Pathol 2018;27:189-197.

10. Williams AR, Ropiak HM, Fryganas C, Desrues O, Mueller-Harvey I,Thamsborg SM. Assessment of the anthelmintic activity of medicinal plant extracts and purified condensed tannins against free-living and parasitic stages of Oesophagostomum dentatum. Parasit Vectors 2014;7:518-527.

11. Klongsiriwet C, Quijada J, Williams AR, Mueller-Harvey I, Williamson EM, Hoste H. Synergistic inhibition of Haemonchus contortus exsheathment by flavonoid monomers and condensed tannins. International J Parasitol Drugs Drug Resist 2015; 5:17-134.

12. Castillo-Mitre GF, Olmedo-Juárez A, Rojo-Rubio R, Cortázar-González M, Mendoza-de Gives P, Hernández-Beteta EE, et al. Caffeoyl and coumaroyl derivatives from Acacia cochliacantha exhibit ovicidal activity against Haemonchus contortus. J Ethnopharmacol 2017;204:125-131.

13. Argueta VA, Cano ALM, Radoarte ME. Atlas de plantas de la medicina tradicional mexicana. México, D.F., 1994.

14. García E. Modificaciones al sistema de clasificación climática de Koeppen. Universidad Nacional Autónoma de México, México. 1987.

15. AOAC (Association of Official Analytical Chemists) Official Methods of Analysis, 16th edition. AOAC, Arlington, VA, USA. 1997.

16. Van Soest PJ, Robertson JB, Lewis BA. Methods for dietary fiber, neutral detergent fiber, and nonstarch polysaccharides in relation to animal nutrition. J Dairy Sci 1991;74:3583-3597.

17. Terrill TH, Rowan AM, Douglas GB, Barry TN. Determination of extractable and bound condensed tannins concentration in forage plants, protein concentrate meals and cereal grains. J Sci Food Agric 1992;58:321-329. 
18. López J, Tejada I, Vásquez C, Garza JD, Shimada A. Condensed tannins in humid tropical fodder crops and their in vitro biological activity: part 1. J Sci Food Agric 2004; 84:291-294.

19. Porter LJ, Hrstich LN, Chan BG. The conversion of procyanidins and prodelphinidins to cyanidin and delhpinidin. Phytochem 1986;25:223-230.

20. Hagerman AE, Butler, LG. In Herbivores: Their interactions with secondary plant metabolites. Volume I. New York: Academic Press; 1991:355-388.

21. Mesquita JR, Mega C, Coelho C, Cruz R, Vala H, Esteves F, et al. ABC series on diagnostic parasitology part 3: The Baermann technique. The Veterinary Nurse 2017;8:10. doi.org/10.12968/vetn.2017.8.10.558

22. Arece J, Mahieu M, Archiméde H, Aumont G, Fernández M, González E, et al. Comparative efficacy of six anthelmintics for the control of nematodes in sheep in Matanzas, Cuba. Small Ruminant Res 2004;5(1-2):61-67.

23. NRC. Nutrient requirements of small ruminants, sheep, goats, cervids, and new world camelids. The National Academy Press, Washington, DC, 2007.

24. SAS Institute SAS User's Guide: Statistics. Ver 9.0. SAS Institute, Cary, N.C., USA, 2006.

25. Steel RGD, Torrie JH. Bioestadística: Principios y procedimientos. McGraw-Hill. México, 1980.

26. Brito DRB, Costa-Júnior LM, Garcia JL, Torres-Acosta JFJ, Louvandini H, Cutrim-Júnior JAA, et al. Supplementation with dry Mimosa caesalpiniifolia leaves can reduce the Haemonchus contortus worm burden of goats. Vet Parsitol 2018;252:47-51.

27. Hoste H, Torres-Acosta JFF, Quijada J, Chan-Pérez I, Dakhel MM, Kommuru DS, et al. Interactions between nutrition and infections with Haemonchus contortus and related gastrointestinal nematodes in small ruminants. Adv Parasitol 2016;93:239-351.

28. Méndez-Ortíz FA, Sandoval-Castro CA, Torres-Acosta JFJ. Short term consumption of Havardia albicans tannin rich fodder by sheep: Effects on feed intake, diet digestibility and excretion of Haemonchus contortus eggs. Anim Feed Sci Technol 2012; 176:185-191.

29. Frutos P, Hervás G, Giráldez FJ, Mantecón AR. Review. Tannins and ruminant nutrition. Span J Agric Res 2004;2:191-202.

30. Waghorn GC, McNabb WC. Consequences of plant phenolic compounds for productivity and health of ruminants. Proc Nut Soc 2003;62:383-392. 
31. Barry TN. Condensed tannins: their role in ruminant protein and carbohydrate digestion and possible effects upon the rumen ecosystem. In: Nolan JV, et al, editors. The roles of protozoa and fungi in ruminant digestion. Armidale, NSW, Australia: Pernambul Books; 1989:153167.

32. León-Castro Y, Olivares-Pérez J, Rojas-Hernández S, Villa-Mancera A, Valencia-Almazán MT, Hernández-Castro E, et al. Effect of three fodders tree on Haemonchus contortus control and weight variations in kids. Eco Rec Agro 2015;2:193-201.

33. Barrau E, Fabre N, Fouraste I, Hoste H. Effect of bioactive compounds from Sainfoin (Onobrychis viciifolia Scop.) on the in vitro larval migration of Haemonchus contortus: role of tannins and flavonol glycosides. Parasitol 2005;31(4):531-538.

34. Brunet $\mathrm{S}$, Hoste $\mathrm{H}$. Monomers of condensed tannins affect the larval exsheathment of parasitic nematodes of ruminants. J Agric Food Chem 2006; 54(20):7481-7487.

35. Quijada J, Fryganas C, Ropiak HM, Ramsay A, Mueller-Harvey I, Hoste H. Anthelmintic activities against Haemonchus contortus or Trichostrongylus colubriformis from small ruminants are influenced by structural features of condensed tannins. J Agric Food Chem 2015;63(28):6346-6354. 